\title{
ESTUDIO COMPARATIVO EN EL MANEJO MÉDICO DEL ABORTO TERAPÉUTICO ENTRE EL USO DE MIFEPRISTONE MÁS MISOPROSTOL Y EL USO DE MISOPROSTOL SOLO
}

\author{
Enrique Guevara Ríos ${ }^{1, a, b}$, Antonio Luna Figueroa ${ }^{1, a, b}$, Miguel Gutiérrez Ramos ${ }^{1,5, a}$, Félix Dasio Ayala- \\ Peralta $^{1 a, b}$, Cesar Carranza Asmat ${ }^{1, a, b}$, Pedro Arango Ochante ${ }^{1, a}$, Marcos Espinola-Sánchez ${ }^{1 c}$, Percy \\ Minaya León ${ }^{1, d}$, Pedro Mascaro Sánchez ${ }^{1, a, b}$, Juan Díaz Villar ${ }^{1, a}$, Felipe Loo Choy ${ }^{1, a}$, Luis Kobayashi \\ Tsutsumi ${ }^{1, a}$, Dennys Celedonio Salvador ${ }^{1, a}$, Luis Almeyda Castro ${ }^{1, a}$, Jhony Juarez Montalvan ${ }^{5}$, \\ Leonardo Lachira León ${ }^{2, a}$, Luis Makiya Onagaa ${ }^{3, a}$, Fredy Jesús Cabrera Olórtegui ${ }^{4, a}$, Melisa Mejico \\ Caja ${ }^{1, e}$, Augusto Racchumí Vela ${ }^{1, f}$.
}

\begin{abstract}
RESUMEN
Objetivos: Evaluar las diferencias en el manejo médico del aborto terapéutico usando Mifepristone más Misoprostol comparado con el uso de Misoprostol solo. Materiales y métodos: Se realizó un estudio observacional, de cohorte retrospectiva y multicéntrica (Instituto Nacional Materno Perinatal [INMP], Hospital Regional de Pucallpa, Hospital Santa Rosa de Piura y Hospital Regional de Loreto), en gestantes con indicación de aborto terapéutico, según lo establecido en la RM N 486-2014-MINSA. Las pacientes enroladas en el INMP, al ingreso recibieron $200 \mathrm{mg}$ de Mifepristona y a las 24 horas Misoprostol $800 \mu \mathrm{g}$ colocado en fondo de saco vaginal posterior al cérvix, seguido de $400 \mu \mathrm{g}$ vía sublingual, cada 3 horas hasta la expulsión del feto y placenta, siendo el tope 5 dosis por día; las pacientes de los tres hospitales restantes se les administro solo Misoprostol bajo el mismo esquema. Resultados: El tiempo de expulsión del feto se consideró desde la administración de la primera dosis del misoprostol hasta las 24 horas; en el grupo de Mifepristona mas Misoprostol fue del $100 \%$ y en el grupo de Misoprostol solo fue del $83 \%(p=0.05)$. La expulsión de la placenta en grupo que uso Mifepristona fue del $96 \%$ y en el grupo de Misoprostol solo fue del $83 \%(p=0.20)$. Se presentaron restos endouterinos en el $10 \%$ del grupo de mifepristona versus $33 \%$ del grupo de solo misoprostol $(p=0.03$ ). El tiempo de expulsión del producto de la concepción fue menor en el grupo de mifepristona con una diferencia de sus medianas de 2.8 horas ( $p$ $=0.001$ ). Conclusiones: Encontramos que existen diferencias en el manejo médico del aborto terapéutico a favor del uso de Mifepristone más Misoprostol versus el uso de Misoprostol solo, en términos de tasa de éxito, tiempo de inducción al aborto y reacciones adversas o complicaciones.
\end{abstract}

Palabras claves: Aborto terapéutico; Aborto Legal; Misoprostol; Mifepristona (Fuente: DeCS BIREME).

\section{COMPARATIVE STUDY IN THE MEDICAL MANAGEMENT OF THERAPEUTIC ABORTION BETWEEN THE USE OF MIFEPRISTONE MISOPROSTOL AND THE USE OF MISOPROSTOL ONLY}

\begin{abstract}
Objectives: To assess the differences in the medical management of therapeutic abortion using Mifepristone plus Misoprostol compared to the use of Misoprostol alone. Materials and methods: An observational, retrospective and multicenter cohort study (National Maternal Perinatal Institute [INMP], Pucallpa Regional Hospital, Santa Rosa de Piura Hospital and Loreto Regional Hospital) was conducted in pregnant women with indication of therapeutic abortion, according to as established in MRI No. 486-2014-MINSA. Patients enrolled in the INMP, upon admission they received $200 \mathrm{mg}$ of Mifepristone and at 24 hours Misoprostol $800 \mu \mathrm{g}$ placed in the bottom of the vaginal sac after the cervix, followed by $400 \mu \mathrm{g}$ sublingually, every 3 hours until the expulsion of the fetus and placenta, the cap being 5 doses per day; the patients of the three remaining hospitals were administered only Misoprostol under the same scheme. Results: The expulsion time of the fetus was considered from the administration of the first dose of misoprostol until 24 hours; in the Mifepristone plus Misoprostol group it was 100\% and
\end{abstract}


in the Misoprostol group it was only $83 \%(p=0.05)$. The expulsion of the placenta in the group that used Mifepristone was $96 \%$ and in the Misoprostol group it was only $83 \%(p=0.20)$. Endouterine remains were present in $10 \%$ of the mifepristone group versus $33 \%$ of the misoprostol-only group $(p=0.03)$. The expulsion time of the product of conception was shorter in the mifepristone group with a difference of their medians of 2.8 hours $(p=0.001)$. Conclusions: We found that there are differences in the medical management of therapeutic abortion in favor of the use of Mifepristone plus Misoprostol versus the use of Misoprostol alone, in terms of success rate, induction time to abortion and adverse reactions or complications.

Keywords: Abortion, Therapeutic; Abortion, Legal; Misoprostol; Mifepristone (Source: MeSH NLM)

\section{INTRODUCCIÓN}

De acuerdo con un estudio de la Organización Mundial de la Salud (OMS) y el Instituto Alan Guttmacher, en el período comprendido entre los años 2010 y 2014 se produjeron en todo el mundo 25 millones de abortos peligrosos (equivalentes al $45 \%$ de todos los abortos) al año. La mayoría de los abortos peligrosos (97\%) se produjo en países en desarrollo de África, Asia y América Latina ${ }^{1}$.

En el Perú, se considera que el Aborto Terapéutico es la interrupción del embarazo hasta las 22 semanas de gestación con el consentimiento de la mujer cuando, el único medio para salvar su vida o para evitar en su salud un mal grave y permanente, es necesaria la interrupción del embarazo. Este procedimiento en el Perú, está normado en la "Guía Técnica Nacional para la estandarización del procedimiento de la Atención Integral de la gestante en la Interrupción Voluntaria por Indicación Terapéutica del Embarazo menor de 22 semanas con consentimiento informado en el marco de lo dispuesto en el artículo $119^{\circ} \mathrm{del}$ Código Penal", y aprobado mediante Resolución Ministerial No 486-2014/MINSA del 27 de junio del $2014^{2,3}$

La provisión de un aborto seguro en toda la extensión de la ley es un componente importante de los servicios de salud reproductiva y difundida por la OMS y la FIGO 4,5 . Esto ha permitido avances en el manejo médico del aborto seguro como alternativa al manejo quirúrgico del mismo. El descubrimiento de la antiprogestina Mifepristona, en la década de 1980, hizo posible desarrollar métodos no quirúrgicos efectivos para la interrupción del embarazo ${ }^{6}$, sin embargo, la evidencia nos muestra que la Mifepristone sola no es suficiente, y el régimen más eficaz y más seguro requiere el uso del Misoprostol, después de la administración de Mifepristona, tanto en el primer trimestre como el segundo trimestre ${ }^{7-9}$. La Organización Mundial de la Salud aprobó tanto la Mifepristone como el Misoprostol para la interrupción del embarazo temprano al incluirlos en la lista de Medicamentos esenciales en 2005 y diferentes organismos de salud internacionales proponen su uso para la culminación del embarazo en condiciones seguras siguiendo pautas claras y bien establecidas ${ }^{5}$.

B. Ganatra y colaboradores ${ }^{10}$, en el estudio citado por la OMS y el Instituto Guttmacher, refieren que "se precisan más esfuerzos, especialmente en las regiones en desarrollo, para garantizar el acceso a los anticonceptivos y al aborto seguro. Cuando las mujeres y las niñas no pueden acceder a servicios eficaces de anticoncepción y aborto seguro, hay graves consecuencias para su propia salud y la de sus familias. Esto no debería pasar. Sin embargo, pese a la evidencia y a los recientes avances en materia de tecnología, siguen produciéndose demasiados abortos peligrosos y demasiadas mujeres continúan sufriendo y muriendo".
El objetivo del presente estudio fue evaluar las diferencias en el manejo médico del aborto terapéutico usando Mifepristone más Misoprostol comparado con el uso de Misoprostol solo, en términos de tasa de éxito, tiempo de inducción al aborto, reacciones adversas o complicaciones y necesidad de legrado uterino o AMEU.

\section{MATERIALES Y MÉTODOS}

Diseño y población de estudio. Se realizó un estudio observacional, de cohorte retrospectiva y multicéntrica en gestantes que tuvieron indicación de aborto terapéutico, según lo establecido en la RM No 486-2014-MINSA, atendidas en los establecimientos de salud participantes: Instituto Nacional Materno Perinatal, Hospital Regional de Pucallpa, Hospital Santa Rosa de Piura y Hospital Regional de Loreto, entre agosto del 2018 y octubre del 2019. Se precisaron 30 participantes en el primer grupo (Mifepristone más Misoprostol) y 30 en el segundo (Misoprostol solo) para detectar como estadísticamente significativa la diferencia entre dos proporciones que tuvieron abortos terapéuticos, para el grupo 1 se estima sea del $96.5 \%$ y el grupo 2 del $76.2 \%$, según Ngoc NT y col ${ }^{7}$.

El muestreo fue por conveniencia, revisando las historias clínicas en forma consecutiva de las gestantes que tuvieron la indicación de aborto terapéutico y que cumplían los criterios de inclusión en el periodo señalado, hasta completar la cuota de muestra calculada para cada grupo. Las pacientes enroladas del INMP, de acuerdo a la "Guía de práctica clínica y procedimientos en obstetricia y perinatología 2018”, al ingreso recibieron 200 mg de Mifepristona y a las 24 horas Misoprostol $800 \mu \mathrm{g}$ colocado en fondo de saco vaginal posterior al cérvix, seguido de $400 \mu \mathrm{g}$ vía sublingual, cada 3 horas hasta la expulsión del feto y placenta, siendo el tope 5 dosis por día, las pacientes de los tres hospitales restantes se les administro solo Misoprostol bajo el mismo esquema ${ }^{11,12}$. Cuando no hubo expulsión del producto con las primeras 5 dosis, se dio un reposo de 12 horas y se continuo con una segunda ronda de Misoprostol con las dosis señaladas.

Técnicas y procedimientos de recolección de datos. La recolección de los datos se realizó revisando las historias clínicas de cada mujer, verificado que hayan tenido seguimiento 7 días posterior al alta, la información fue descargada en la ficha de recolección e ingresada a un formulario electrónico, por un personal entrenado externo a la investigación, posteriormente dichos datos fueron exportados a una hoja de cálculo de Excel para la generación de la base de datos.

En la construcción de la base de datos se garantizó la confidencialidad de la identidad de las participantes, identificando a cada la paciente con un código conocido solo por los investigadores. El investigador responsable 
de cada sede hospitalaria participante realizó el monitoreo de la ejecución y recolección de datos.

Análisis de datos. Consistió en la segmentación en grupos comparativos a los cuales se les realizó las descripciones bivariadas de los datos, tanto a las de naturaleza cuantitativa como cualitativas. Se aplicó resúmenes de frecuencia absolutas y porcentuales, y medidas de comparación proporcional en función a chi cuadrado para las variables categóricas; mientras que, para las variables cuantitativas se utilizó medidas de posición central y de variabilidad, así como la aplicación de estimaciones interválicas, garantizando la pertenencia del parámetro población de las variables de cada unidad de análisis. Las pruebas inferenciales utilizadas fueron Chi cuadrado y la prueba de rangos $U$ de Mann-Whitney; el valor crítico para la toma de decisión estadística se estableció un nivel de significancia del 0.05 y un nivel de confianza del $95 \%$. El procesamiento de los datos fue realizado en la hoja de cálculo Excel y el software estadístico R versión 3.6.

Consideraciones éticas. Tomamos como fuente de información, la revisión de las historias clínicas de los casos seleccionados, por lo que no se requirió el consentimiento informado, la identificación de las pacientes fueron codificadas para poder proteger su privacidad y confidencialidad. Así mismo, el protocolo de investigación fue evaluado y aprobado por el Comité de Ética del INMP y se solicitó la aprobación de la máxima autoridad de cada establecimiento de salud participante.

\section{RESULTADOS}

El grupo de estudio estuvo compuesto por 60 pacientes, cuyas características sociodemográficas se describe en la tabla 1. La población estuvo conformada principalmente por multíparas, el rango de edad predominante fue de 20 a 34 años con un $70 \%$.

La edad gestacional al momento de la interrupción del embarazo en toda la muestra en un $54 \%$ fue $\geq$ a 18 semanas, la mediana de en el grupo del INMP fue de 18 semanas con un mínimo de 11ss y un máximo de 21ss, el $66,7 \%$ correspondió a una edad gestacional $\geq$ a 17 semanas; En el grupo de otros hospitales la mediana fue de 19 semanas con un mínimo de 7 semanas y un máximo de 21 semanas, el $66,7 \%$ correspondió a una edad gestacional $\geq$ a 19 semanas.

Tabla 1: Características Socio demográficas de las participantes

\begin{tabular}{|c|c|c|c|c|c|c|}
\hline \multicolumn{2}{|c|}{ Características } & \multicolumn{2}{|c|}{$\begin{array}{c}\text { INMP } \\
\text { (Mife +Miso) }\end{array}$} & \multicolumn{2}{|c|}{$\begin{array}{c}\text { Otros hospitales } \\
\text { (Solo Miso) }\end{array}$} & \multirow{2}{*}{$\begin{array}{c}\text { Chi-cuadrado } \\
\text { P }\end{array}$} \\
\hline & & $\mathbf{N}$ & $\%$ & $\mathbf{N}$ & $\%$ & \\
\hline \multirow{4}{*}{$\begin{array}{c}\text { Rango de edades de } \\
\text { mujeres }\end{array}$} & 10 a 19 años & 4 & $13.3 \%$ & 4 & $13.3 \%$ & 1 \\
\hline & 20 a 34 años & 21 & $70.0 \%$ & 20 & $66.7 \%$ & 1 \\
\hline & 35 a más & 5 & $16.7 \%$ & 6 & $20.0 \%$ & 1 \\
\hline & Total & 30 & $100.0 \%$ & 30 & $100.0 \%$ & \\
\hline \multirow{5}{*}{ Grado de instrucción } & Analfabeta & 0 & $0.0 \%$ & 2 & $6.7 \%$ & - \\
\hline & Primaria & 3 & $10.0 \%$ & 1 & $3.3 \%$ & 0.61 \\
\hline & Secundaria & 15 & $50.0 \%$ & 20 & $66.7 \%$ & 0.30 \\
\hline & Superior & 12 & $40.0 \%$ & 7 & $23.3 \%$ & 0.27 \\
\hline & Total & 30 & $100.0 \%$ & 30 & $100.0 \%$ & \\
\hline \multirow{4}{*}{ Seguro de salud } & No tiene & 3 & $10.0 \%$ & 0 & $0.0 \%$ & - \\
\hline & EsSalud & 3 & $10.0 \%$ & 2 & $6.7 \%$ & 1 \\
\hline & SIS & 24 & $80.0 \%$ & 28 & $93.3 \%$ & 0.26 \\
\hline & Total & 30 & $100.0 \%$ & 30 & $100.0 \%$ & \\
\hline \multirow{4}{*}{ Paridad } & Multipara (2 o más hijos) & 13 & $43.3 \%$ & 15 & $50.0 \%$ & 0.80 \\
\hline & Nulípara (0 hijos) & 10 & $33.3 \%$ & 9 & $30.0 \%$ & 1 \\
\hline & Primípara (1 hijo) & 7 & $23.3 \%$ & 6 & $20.0 \%$ & 1 \\
\hline & Total & 30 & $100.0 \%$ & 30 & $100.0 \%$ & \\
\hline \multirow{5}{*}{$\begin{array}{l}\text { Hospital de } \\
\text { procedencia }\end{array}$} & INMP & 30 & $100.0 \%$ & 0 & $0.0 \%$ & - \\
\hline & Iquitos - Loreto & 0 & $0.0 \%$ & 9 & $30.0 \%$ & - \\
\hline & Pucallpa - Ucayali & 0 & $0.0 \%$ & 7 & $23.3 \%$ & - \\
\hline & Santa Rosa - Piura & 0 & $0.0 \%$ & 14 & $46.7 \%$ & - \\
\hline & Total & 30 & $100.0 \%$ & 30 & $100.0 \%$ & \\
\hline
\end{tabular}


El diagnóstico principal para la interrupción terapéutica del embarazo (tabla 2) fue "otra patología materna que ponga en riesgo la vida de la gestante o genere en su salud un mal grave y permanente" con $85 \%$ y significativamente más casos en el INMP (97\%) que en otros hospitales $(73 \%)$ con un $p=0,03$.

Tabla 2. Diagnósticos por los cuales se realizó el aborto terapéutico

\begin{tabular}{|c|c|c|c|c|c|c|c|}
\hline \multirow{2}{*}{ Diagnóstico } & \multicolumn{2}{|c|}{ Total } & \multicolumn{2}{|c|}{ INMP } & \multicolumn{2}{|c|}{ Otros hospitales } & \multirow{2}{*}{$\begin{array}{c}\text { Chi-cuadrado } \\
\text { P }\end{array}$} \\
\hline & $\mathbf{n}$ & $\%$ & $\mathbf{n}$ & $\%$ & $\mathbf{n}$ & $\%$ & \\
\hline $\begin{array}{l}\text { Otra patología materna que ponga en riesgo } \\
\text { la vida de la gestante o genere en su salud } \\
\text { un mal grave y permanente. }\end{array}$ & 51 & $85.0 \%$ & 29 & $96.7 \%$ & 22 & $73.3 \%$ & 0.03 \\
\hline Insuficiencia cardiaca congestiva. & 3 & $5.0 \%$ & 1 & $3.3 \%$ & 2 & $6.7 \%$ & $>0.05$ \\
\hline $\begin{array}{l}\text { Lupus Eritematoso Sistémico con daño renal } \\
\text { severo refractario a tratamiento, Insuficiencia } \\
\text { respiratoria severa. }\end{array}$ & 3 & $5.0 \%$ & 0 & $0.0 \%$ & 3 & $10.0 \%$ & - \\
\hline $\begin{array}{l}\text { Neoplasia maligna que requiera tratamiento } \\
\text { quirúrgico. }\end{array}$ & 1 & $1.7 \%$ & 0 & $0.0 \%$ & 1 & $3.3 \%$ & - \\
\hline Hiperémesis gravídica refractaria. & 2 & $3.3 \%$ & 0 & $0.0 \%$ & 2 & $6.7 \%$ & - \\
\hline Total & 60 & $100.0 \%$ & 30 & $100.0 \%$ & 30 & $100.0 \%$ & \\
\hline
\end{tabular}

El tiempo de expulsión del feto se consideró desde la administración de la primera dosis del misoprostol hasta las 24 horas, en grupo de Mifepristona mas Misoprostol fue de $100 \%$ y en el grupo de Misoprostol solo fue $83 \%$ $(p=0.05)$. El tiempo de expulsión del producto de la concepción fue menor en el grupo de mifepristona con una diferencia de sus medianas de 2.8 horas ( $p=0.001$ ) y se detalla en la gráfica 1 . El $87 \%$ del grupo de INMP expulso el producto con 2 dosis de Misoprostol, para el grupo control fue $43 \%$ (tabla 3 ).

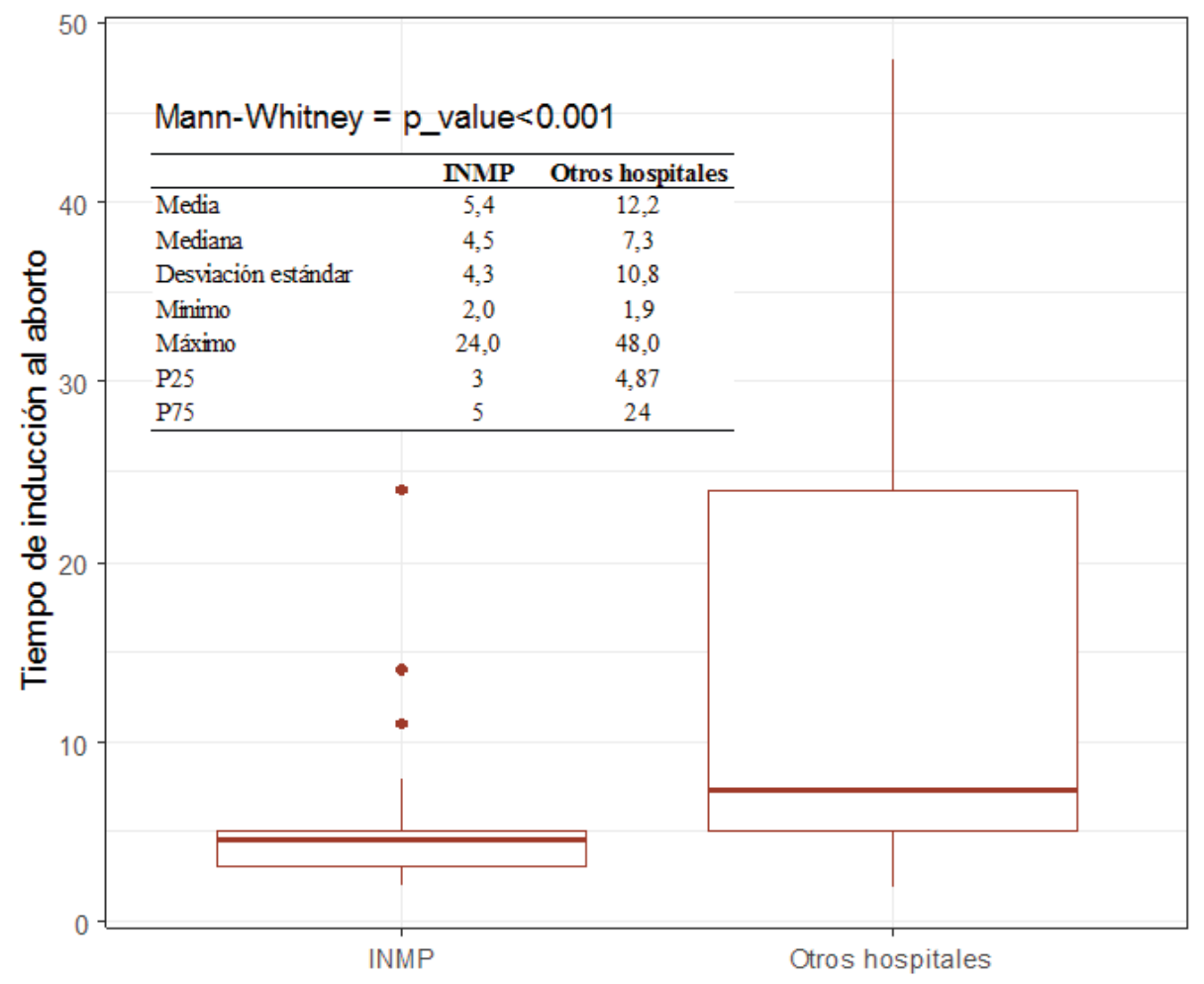

La expulsión de la placenta en grupo que uso Mifepristona fue del $96 \%$ y en el grupo de Misoprostol solo fue $83 \%$ ( $p$ $=0.20$ ). Se presentaron restos endouterinos en el $10 \%$ del grupo de mifepristona versus $33 \%$ del grupo de solo misoprostol $(p=0.03)$. El sangrado leve fue mayor en el grupo de mifepristona con un $96 \%$ en comparación del grupo de solo misoprostol con un $80 \%(p=0.11)$. Se realizó AMEU/legrado en el $96 \%$ del grupo de Mifepristona y $93 \%$ en el de solo Misoprostol $(p=1)$. 
Tabla 3. Numero de dosis administrada de misoprostol

\begin{tabular}{cccccccc}
\hline $\begin{array}{c}\text { Número de dosis } \\
\text { administrada }\end{array}$ & \multicolumn{2}{c}{ Total } & \multicolumn{2}{c}{ INMP } & \multicolumn{2}{c}{ Otros hospitales } & \multicolumn{2}{c}{ Chi cuadrado } \\
\hline 1 & $\mathbf{n}$ & $\mathbf{n}$ & $\mathbf{n}$ & $\mathbf{n}$ & $\mathbf{0}$ & $\mathbf{p}$ \\
\hline 2 & 15 & $25.0 \%$ & 10 & $33.3 \%$ & 5 & $16.7 \%$ & 0,233 \\
\hline 3 & 24 & $40.0 \%$ & 16 & $53.3 \%$ & 8 & $26.7 \%$ & 0,065 \\
\hline 4 & 7 & $11.7 \%$ & 1 & $3.3 \%$ & 6 & $20.0 \%$ & 0,107 \\
\hline 5 & 2 & $3.3 \%$ & 1 & $3.3 \%$ & 1 & $3.3 \%$ & 1 \\
\hline$>5$ & 11 & $18.3 \%$ & 2 & $6.7 \%$ & 9 & $30.0 \%$ & 0,045 \\
\hline Total & 1 & $1.7 \%$ & 0 & $0 \%$ & 1 & $3.3 \%$ & - \\
\hline
\end{tabular}

Las reacciones observadas con el uso de la mifepristona fue el sangrado escaso en el 33\% y nauseas en el 3\%, no se observaron otras reacciones en relación con este medicamento, con respecto al uso del Misoprostol fue el sangrado escaso $88 \%$, seguido de dolor pélvico $38 \%$, nauseas $7 \%$, fiebre $5 \%$, diarrea $3 \%$ y otras reacciones adversas $5 \%$. El sangrado moderado - severo de ambos grupos fue $12 \%$ en promedio, siendo en el grupo de solo Misoprostol un $20 \%$ versus un $3 \%$ en la combinación $(p=0,04)$.

La presencia de restos endouterinos en el grupo de mifepristona fue del $10 \%$ y en el grupo de solo misoprostol fue $33 \%$ ( $p=0,028)$, el AMEU / legrado uterino fue realizado en el $96 \%$ del grupo de mifepristona y $93 \%$ en el de solo misoprostol $(p=0,554)$.

\section{DISCUSIÓN}

El Instituto Nacional Materno Perinatal como ente rector en la salud de la mujer y la prevención de la morbimortalidad materna y perinatal, brinda de manera protocolizada el manejo médico y quirúrgico del Aborto Terapéutico en gestantes que lo solicitan y cumplen con los criterios establecidos según norma técnica.

De acuerdo a los resultados, ambos grupos de estudio fueron comparables, la necesidad de un aborto terapéutico ha estado presente, principalmente en mujeres multíparas (43\% en Mifepristona y $50 \%$ en Misoprostol), con un rango de edad de 20 a 34 años $(70 \%$ en Mifepristona y $67 \%$ en Misoprostol), sin embargo, vale la pena anotar que el grupo de 10 a 19 años de edad son el $13 \%$ en el grupo de Mifepristona y el $13 \%$ en el de Misoprostol, esto crea la necesidad de profundizar con otras investigaciones que enfoquen el grupo entre 10 y 15 años. Los diagnósticos que han permitido a las mujeres poner en práctica su derecho a la realización del aborto terapéutico fueron el de "otra patología materna que ponga en riesgo la vida de la gestante o genere en su salud un mal grave y permanente", que aparece con el número 11 de la Guía Nacional ( $85 \%$ del total), seguido de insuficiencia cardiaca congestiva y lupus que suman un $10 \%$, similares resultados encontrado por Guevara el 2015, en un estudio retrospectivo donde revisó todos los casos de interrupción terapéutica del embarazo realizados en el INMP entre los años 2009 y el 2013, donde reporto que la principal indicación de interrupción de embarazo fue el riesgo de salud materna con un $87 \%$ de los casos revisados ${ }^{13}$.

Este resultado ratifica algo que los propios profesionales vienen planteando, la necesidad de revisar los diagnósticos contenidos en la Guía Nacional para su ampliación.

El tiempo de expulsión del producto de la concepción fue menor en el grupo de mifepristona con una diferencia de sus medianas de 2,8 horas ( $p<0.001$ ), en concordancia con otros estudios ${ }^{14}$ y la propuesta de la OMS. Carbonell en un estudio realizado el 2001 en Valencia - España, donde estudio a 450 mujeres con indicación de aborto en el primer trimestre encontró que el tiempo promedio de expulsión fue de $4,1 \pm 2,1 \mathrm{~h}{ }^{15}$. La expulsión del feto dentro de las primeras 24 horas, en el grupo que recibió Mifepristona mas Misoprostol fue de $100 \%$, mientras que en el grupo que sólo recibió Misoprostol solo fue $83 \%$ $(p=0.05)$, concordante con Akkenapally ${ }^{16}$ que el 2016 realizo un estudio en la India similar al nuestro con 200 mujeres, encontrando que la tasa de éxito en el Grupo I (solo Misoprostol) fue del 89\%, mientras que en el Grupo II (Misoprostol mas mifepristona) fue del 96\%, mientras que en el 2017 Louie et al. en Armenia reportaron una tasa de expulsión de 99,2\%, que es muy cercana a la nuestra ${ }^{17}$. Este resultado se encuentra relacionado con el número de dosis utilizadas de Misoprostol, donde se observa una tendencia de más dosis a los que no usaron previamente mifepristona, en el grupo del INMP más del doble de pacientes ya habían expulsado el producto con dos dosis ( $87 \%$ versus el $43 \%)$.

De acuerdo con los resultados, el sangrado leve fue mayor en el grupo de mifepristona con un $96 \%$ en comparación del grupo de misoprostol con un $80 \%$ ( $p=0.044)$, debemos anotar que el sangrado pos intervención moderado severo fue mayor en el grupo de misoprostol con un $20 \%$ versus un $3 \%$ en el grupo de Mifepristona - Misoprostol $(p=0.04)$, una revisión hecha por Sitruk-Ware en el 2006 encontró que el sangrado excesivo fue del $10 \%$ en la pacientes que usaron mifepristona previamente mayor a lo reportado por nuestro estudio ${ }^{18}$. La presencia de 
restos endouterinos en el grupo de mifepristona fue del $10 \%$ y en el grupo de solo misoprostol fue $33 \%$ ( $p=$ 0.03). La realización de AMEU/legrado estuvo presente en el $96 \%$ del grupo de mifepristona y $93 \%$ en el de solo misoprostol ( $p>0.05$ ), aparentemente y de acuerdo a lo manifestado por los profesionales, la realización de AMEU/Legrado, en la mayoría de los casos es por "la necesidad de confirmar que no han quedado restos", similar conducta reportada por Chai en el 2009 donde las pacientes reciben dilatación y legrado de forma rutinaria después del aborto con medicamentos ${ }^{19}$.

Las reacciones observadas con la Mifepristona fueron escasas, sangrado escaso en el $33 \%$ y nauseas en el $3 \%$, en el caso del misoprostol se han mostrado otros efectos como el sangrado moderado severo que fue mayor en el grupo de solo Misoprostol con una diferencia significativa $(p=0,04)$, no se encontró diferencia significativa en ambos grupos con respecto al dolor pélvico $(38 \%)$, náuseas $(7 \%)$, fiebre $(5 \%)$, diarrea $(3 \%)$, similar a otros estudios los síntomas más importantes están relacionadas al uso de Misoprostol, pero en nuestro caso los porcentajes fueron menores, en una revisión sistemática de Chen y Creinin reportaron que la ocurrencia de nauseas fue (34 - 69\%), fiebre (12 - 48\%) y diarrea(2-61\%) ${ }^{20}$. El uso de Mifepristona para el manejo médico del Aborto Terapéutico, a pesar de toda la evidencia existente ${ }^{18,21-23}$, no se encuentra dentro de las guías clínicas de todos los establecimientos de salud, que ofrecen dicho servicio, por lo que proponemos una mayor difusión de su eficacia y seguridad que permita extender su uso.

Las limitaciones del presente estudio son las derivadas de un estudio retrospectivo, el tipo de muestreo y el tamaño de muestra, pero consideramos que, al ser el primer estudio nacional, con el uso de mifepristona nos da una información base para poder desarrollar estudios prospectivos y poder contar prontamente con este medicamento, como un aliado terapéutico de fácil acceso.

En conclusión, los resultados que estamos presentando nos permiten afirmar que Existen diferencias del manejo médico del aborto terapéutico usando mifepristone más misoprostol comparado con el uso de misoprostol solo. Este estudio de investigación ha demostrado que, si existen diferencias en el manejo médico del aborto terapéutico usando mifepristone más misoprostol comparado con el uso de misoprostol solo, siendo favorables a la combinación, en términos de tasa de éxito, tiempo de inducción al aborto y reacciones adversas o complicaciones.

Financiamiento: En parte por Patfhinder International sede en Perú.

Conflicto de interés: Los autores declaran no tener algún conflicto de intereses.

\section{REFERENCIAS BIBLIOGRÁFICAS}

1. Sedgh G, Bearak J, Singh S, Bankole A, Popinchalk A, Ganatra B, et al. Abortion incidence between 1990 and 2014: global, regional, and subregional levels and trends. The Lancet. 16 de julio de 2016;388(10041):258-67.
2. Aspilcueta Gho D, Ramos Chavez I. Proceso de aprobación e implementación de la «Guía Técnica Nacional para la Estandarización del Procedimiento de la Atención Integral de la Gestante en la Interrupción Voluntaria por Indicación Terapéutica del Embarazo menor de 22 Semanas con Consentimiento Informado en el marco de lo dispuesto en el Artículo $119^{\circ}$ del Código Penal»: Para atender casos de aborto por causal salud o terapéutico en el Perú. Anales de la Facultad de Medicina. octubre de 2015;76(4):397-406.

3. MINSA. Resolución Ministerial $N^{\circ}$ 486-2014-MINSA [Internet]. 2014 [citado 10 de diciembre de 2019]. Disponible en: https://www.gob.pe/institucion/minsa/ normas-legales/198435-486-2014-minsa

4. Faúndes A. The combination of mifepristone and misoprostol for the termination of pregnancy. International Journal of Gynecology \& Obstetrics. 2011;115(1):1-4.

5. Organization WH. Medical Management of Abortion [Internet]. 2018. ${ }^{a}$ ed. Geneva: World Health Organization; 2019. 1-3 p. Disponible en: https://www.who.int/ reproductivehealth/publications/medical-managementabortion/en/

6. Davey A. Mifepristone and prostaglandin for termination of pregnancy: contraindications for use, reasons and rationale. Contraception. 1 de julio de 2006;74(1):16-20.

7. Ngoc NTN, Blum J, Raghavan S, Nga NTB, Dabash $\mathrm{R}$, Diop A, et al. Comparing two early medical abortion regimens: mifepristone+misoprostol vs. misoprostol alone. Contraception. 1 de mayo de 2011;83(5):410-7.

8. Shaw KA, Topp NJ, Shaw JG, Blumenthal PD. Mifepristone-Misoprostol Dosing Interval and Effect on Induction Abortion Times: A Systematic Review. Obstetrics \& Gynecology. junio de 2013;121(6):1335.

9. Raymond EG, Shannon C, Weaver MA, Winikoff B. Firsttrimester medical abortion with mifepristone $200 \mathrm{mg}$ and misoprostol: a systematic review. Contraception. 1 de enero de 2013;87(1):26-37.

10. Ganatra B, Gerdts C, Rossier C, Johnson BR, Tunçalp Ö, Assifi A, et al. Global, regional, and subregional classification of abortions by safety, 2010-14: estimates from a Bayesian hierarchical model. The Lancet. 25 de noviembre de 2017;390(10110):2372-81.

11. Instituto Nacional Materno Perinatal. Guías de práctica Clínica y de procedimientos en obstetricia y perinatología [Internet]. 2. ${ }^{\text {a }}$ ed. Lima - Perú: INMP; 2018. 534 p. Disponible en: https://www.inmp.gob.pe/institucional/ publicaciones/1439919401

12. OMS. Manual de práctica clínica para un aborto seguro [Internet]. Montevideo, Uruguay: World Health Organization; 2014. 64 p. (NCBI Bookshelf). Disponible en: https://www.who.int/reproductivehealth/publications/ unsafe_abortion/clinical-practice-safe-abortion/es/

13. Guevara Ríos E. Interrupción terapéutica del embarazo en el Instituto Nacional Materno Perinatal , Perú, 2009-2013. Revista Peruana de Ginecología y Obstetricia. enero de 2016;62(1):19-30.

14. Dabash R, Chelli H, Haji S, Shochet T, Raghavan S, Winikoff B. A double-blind randomized controlled trial of mifepristone or placebo before buccal misoprostol for abortion at 14-21 weeks of pregnancy. International Journal of Gynecology \& Obstetrics. 2015;130(1):40-4.

15. Carbonell JL, Varela L, Marí J, Salvador I, Aragón S, Guillem S, et al. Mifepristona (600 mg) + misoprostol $(400 \mu \mathrm{g})$ en el aborto del primer trimestre. Progresos de Obstetricia y Ginecología. 1 de enero de 2001;44(9):361-7.

16. Akkenapally PL. A Comparative Study of Misoprostol Only and Mifepristone Plus Misoprostol in Second Trimester Termination of Pregnancy. J Obstet Gynecol India. 1 de octubre de 2016;66(1):251-7. 
17. Louie KS, Chong E, Tsereteli T, Avagyan G, Abrahamyan $R$, Winikoff B. Second trimester medical abortion with mifepristone followed by unlimited dosing of buccal misoprostol in Armenia. The European Journal of Contraception \& Reproductive Health Care. 2 de enero de 2017;22(1):76-80.

18. Sitruk-Ware R. Mifepristone and misoprostol sequential regimen side effects, complications and safety. Contraception. 1 de julio de 2006;74(1):48-55.

19. Chai J, Tang OS, Hong QQ, Chen QF, Cheng LN, Ng E, et al. A randomized trial to compare two dosing intervals of misoprostol following mifepristone administration in second trimester medical abortion. Hum Reprod. 1 de febrero de 2009;24(2):320-4.

20. Chen MJ, Creinin MD. Mifepristone With Buccal Misoprostol for Medical Abortion: A Systematic Review. Obstetrics \& Gynecology. julio de 2015;126(1):12.

21. Gatter M, Cleland K, Nucatola DL. Efficacy and safety of medical abortion using mifepristone and buccal misoprostol through 63 days. Contraception. 1 de abril de 2015;91(4):269-73.
22. Goldstone P, Walker C, Hawtin K. Efficacy and safety of mifepristone-buccal misoprostol for early medical abortion in an Australian clinical setting. Australian and New Zealand Journal of Obstetrics and Gynaecology. 2017;57(3):366-71.

23. DeHart RM, Morehead MS. Mifepristone: Annals of Pharmacotherapy [Internet]. 28 de junio de 2016 [citado 10 de diciembre de 2019]; Disponible en: https://journals. sagepub.com/doi/pdf/10.1345/aph.10397

\section{Correspondencia:}

Enrique Guevara Ríos

Dirección: Jr. Santa Rosa N 941-Cercado de Lima. Correo electrónico: enriqueguevararios@gmail.com Teléfono: 992767453 